\section{Atlas of Breast Surgery}

ISMAIL JATOI, MANFRED KAUFMANN, JEAN PETIT

\begin{tabular}{ll}
\hline EXTENT P/H & $133 \mathrm{p}$, hardback \\
PRICE/ISBN & $£ 100.00 \quad$ 3540243518 \\
PUBLISHER & Springer (Berlin), 2005 \\
REVIEWER & Hugh Bishop \\
STAR RATING & $* * * *$
\end{tabular}

This is a practical and convenient atlas of breast surgery. The authors are well recognised in their field, with a considerable reputation for oncoplastic breast surgery. Unlike many atlases of surgery, this is a slim and convenient volume to carry in a briefcase.

Does it fulfil its promise? The answer is yes: mostly. Let us deal first with the weaker parts. In the seven chapters in this atlas, one is devoted to diagnostic procedures. I cannot see the relevance of this chapter to a breast surgeon as the book is described as an atlas of breast surgery not a handbook for radiologists. Furthermore, the description of how breast abscesses are drained reflects a style of practice that is less often seen these days in the NHS. While some breast abscesses will always need incision and drainage, the majority can be managed by repeated ultrasound aspiration.

I so enjoyed the historical section and appreciated the chapter that contained a photograph of the late and muchlamented John Bostwick, who did so much to advance and teach oncoplastic techniques.

The basis of any surgical procedure is anatomy and the chapter dealing with this is pertinent and pithy even if the section on the lymphatic drainage of the breast has been transferred to the section on axillary surgery. There are good practical tips for the surgical trainee with numerous line drawings. In particular, there are excellent diagrams for the placing of incisions on the breast.

Some of the incisions for approaching gynaecomastia were new to me and, in addition, the authors also stress that excision of a gynaecomastia is often combined with liposuction. The photograph of a disastrous aesthetic outcome from poorly thought-out breast-conserving surgery, one of the few photographs in the book, is a stark reminder that poorly planned conservation surgery can be associated with a truly dreadful aesthetic result. The helpful photograph beneath shows the incisions needed to avoid an ugly divot in the breast. There are also useful hints on how to place incisions for the patient who needs a mastectomy but whose tumour is eccentrically placed in the breast.

The description of the incisions needed to achieve a reasonable aesthetic result in breast-conserving surgery is excellent and includes a good series of illustrations demonstrating the mammoplastic technique. The final chapter on plastic and reconstructive surgery is excellent, with clear diagrams and lots of useful tips for both trainees and the more experienced.

This is an atlas and so one can forgive the fact that indications and contra-indications are omitted. The authors assume that the reader has enough knowledge to match the right operation to the right patient. Indeed, there is no mention of any complications. More importantly, I would have appreciated tips on how to avoid trouble or where the procedure is most at risk. The authors assume that the surgeon has an excellent anaesthetist who can perfuse a patient's brain and also the flap they have dissected without damage. In addition, some thoughts on the management of flaps postoperatively would have been useful.

What would I add to a future addition? Not much. I would remove the chapter on diagnostic procedures, substituting a chapter on marking up a patient pre-operatively and include line diagrams of how that often difficult process is achieved. Finally, trainees frequently find measuring a patient for an implant difficult. I would include a chapter on this in a future edition.

This is a useful book to have in theatre or out-patients and I recommend it.

\section{Understanding Doctors' Performance}

JIM COX, JENNIFER KING, ALLEN HUTCHINSON, PAULINE MCAVOY

$\begin{array}{ll}\text { EXTENT P/H } & 184 \mathrm{p}, \text { paperback } \\ \text { PRICE/ISBN } & £ 27.95 \quad 1857757661 \\ \text { PUBLISHER } & \text { Radcliffe (Oxford), 2005 } \\ \text { REVIEWER } & \text { Tony Giddings } \\ \text { STAR RATING } & * * *\end{array}$

This book is a scholarly and concise review of the evidence so far. This shows a disturbing picture of committed professionals experiencing disproportionate stress and failure in a hostile workplace. The book is short, well written and authoritative. Many chapters are a model of the painstaking evaluation of evidence. Particularly useful are those on stress and depression (including some reference to organisational causes), the psychological factors linked to performance and the account of workload, sleep loss and shift work.

Perhaps not surprisingly in a first edition, it has not ventured into detailed consideration about the defects in training and organisation that have produced such a challenging workplace and poor record of caring for the vulnerable employee. There is evidence that working for the NHS carries a higher risk of personal stress and failure than other British institutions and that depression and suicide are uncomfortably common especially in women.

This is of particular concern at a time when the feminisation of the work-force proceeds apace and our dependence on women in medicine is growing and irreversible. It is perhaps 
most worrying that the book leaves us with the clear impression that a profession that should be most focused on the care of others has developed a culture in which the way that doctors treat their colleagues leaves so much room for improvement. There are serious concerns here about the nature of true professionalism because of the well-documented prevalence of interpersonal disputes, ineffective team-working, bullying and harassment.

Recent work by the General Medical Council, royal colleges, the National Clinical Assessment Service and deaneries has shown that the general levels of skill and understanding required by consultant surgeons, trainers and trusts are worryingly low. There are serious implications here for patient safety not only because of individual failure but because those failures put vulnerable patients at even greater risk.

Since the events at Bristol more than a decade ago, much has been learned about doctors' performance but much less about the causes and opportunities for improvement. Like other industries, about $6 \%$ of the work-force will show significant performance concerns in any one year but health professionals are particularly at risk because of the sensitivity of their work, the variety of problems they face and the poor standard of support in the NHS today.

Repeatedly, the key features of dysfunctional NHS practice are brought into focus. These include the illusion of independent practice and the inappropriate dependence on trainees to provide the service. We should be concerned that, after many years of training, surgical consultants often lack the skills to develop effective team-working and leadership.

Overall, therefore, the book raises more questions than it answers. Perhaps the most important of these is why so little of the effort that has been directed to exposing poor performance and its management has been redirected to improving the chaotic and arbitrary environment in which so many doctors have to work. This book is strong on symptoms. I hope that the next edition will also look at the underlying political and organisational causes and the opportunities for improvement.

\section{Clinical Evaluation of Medical Devices: Principles and Case Studies (2nd edn)}

KAREN BECKER, JOHN WHYTE

\begin{tabular}{ll}
\hline EXTENT P/H & $360 \mathrm{p}$, hardback \\
PRICE/ISBN & $£ 85.50 \quad 1588294226$ \\
PUBLISHER & Humana (New Jersey), 2005 \\
REVIEWER & John Williams \\
STAR RATING & $* *$
\end{tabular}

From the outset, it must be made clear that this book has been written entirely for the American market and hence deals with American legislation.
First published in 1997, rapid development of medical devices has stimulated the need for a second edition. Bearing in mind that the American legislation and workings of the Food and Drug Administration (FDA) are quite different from those encountered in the UK (where the responsible body is the Medicines and Healthcare products Regulatory Agency), the contributors are all North American apart from one Norwegian.

Despite this, there are as always chapters that are worthy of note, some of which are particularly helpful. Chapter 1 deals entirely with clinical trials and having dealt with definition of a medical device then provides an important overview of considerations to be taken into account when organising an appropriate clinical trial. This is a helpful section with a lot of practical advice on the standards required and the sources of potential hazards and pitfalls. It looks to pre-market testing, clinical outcomes and the postmarket surveillances while addressing the statutory requirements of the FDA.

The debate as to whether emerging technologies are covered by the medicines or the devices side of the Agency is addressed in a single chapter. This is not an unfamiliar situation and is becoming an increasingly pointed debate in the UK. As such, the role of the National Institute for Health and Clinical Excellence raised within this chapter does give some indication of the attempt to look internationally. The second part of the chapter largely covers cost, which is of no direct relevance to the UK situation.

Chapter 4 exposes the difference between the British and American systems, with the UK singularly focused on postmarketing surveillance. However, the reporting systems do appear to be markedly similar.

Chapter 5 is especially useful, looking at the statistics associated with the Bayesian method in clinical trials. This fundamental mathematical approach to clinical trials answers many of the difficulties associated with small trial numbers, particularly with multiple end-points.

The development of emerging technologies and the combination of drug/device combinations is examined in chapter 9. Many of the issues discussed, particularly the nature of principal action of the device, are looked at in detail. Systematic exposure to drugs from devices such as drug-eluting stents and the discussion on their toxicology (and hence their relevance as a device or a medicine) are illustrated. Chapter 10 is especially interesting and revealing as it discusses the issues of the production of new devices on the stock market: a sensitive area often overlooked.

Part 2 of the book deals with a series of clinical examples. As such it is particularly useful, covering many of the problems anyone involved in the development of medical devices will encounter, for example, the use of randomised controlled trials and how difficult it is to apply them to devices. 